\title{
Mitochondrial-Targeted Antioxidants Given at Reperfusion Protect Cardiac and Hindlimb Muscles against Ischemia/Reperfusion Injury
}

Keywords: Myocardial ischemia/reperfusion injury; Hindlimb ischemia/ reperfusion; Mitochondrial antioxidants; Nitric oxide; Hydrogen peroxide

\begin{abstract}
Background and purpose: The principal cause of cardiomyocyte dysfunction and necrosis resulting from Ischemia/Reperfusion (I/R) injury is the production and release of Reactive Oxygen Species (ROS) from damaged mitochondria. Mitochondrial ROS-mediated oxidation of cellular proteins and lipids disrupts both cellular metabolism and organelle integrity, leading to depletion of ATP stores and an increase in intracellular calcium ( $\mathrm{Ca}^{2+}$ ) levels. Thus, attenuation of I/R-induced ROS production has been a therapeutic strategy to salvage damaged cardiomyocytes and thereby limit cardiac functional impairment and infarct size [1]. Previous studies have tested the effectiveness of mitochondrial-targeted antioxidants. Mitoquinone (MitoQ) was effective in reducing I/R injury when given only prior to prolonged ischemia, while Szeto-Schiller (SS)-31 was effective when given either prior to ischemia or at the beginning of reperfusion. This study was undertaken to determine whether these agents are effective in limiting myocardial and hindlimb I/R injury when given during the first $5 \mathrm{~min}$ of reperfusion only, and thereby support the premise that mitochondrial damage underlies reperfusion-induced cell death during the initial minutes of reperfusion.
\end{abstract}

Experimental approach: Male Sprague-Dawley rats (275-325 g) were randomized into myocardial or hindlimb I/R groups. Isolated, retrogradely perfused hearts were subjected to global I(30 $\mathrm{min}) / \mathrm{R}(45$

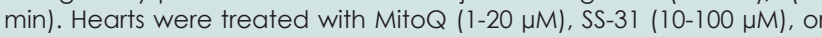
plasma (control) added to the perfusate at the onset of reperfusion and was assessed for cardiac function and infarct size. In the hindlimb experiments, either hydrogen peroxide $\left(\mathrm{H}_{2} \mathrm{O}_{2}\right)$ or Nitric Oxide (NO) sensors were placed randomly in both the right and left femoral veins of the same animal. One limb was subjected to I(30 min)/R(45 min) by reversibly clamping the femoral artery/vein, while the other limb served as a sham. The animal received an intravenous (i.v.) bolus of either MitoQ (1.8 $\left.\mathrm{mg} \mathrm{kg}^{-1}\right)$, SS-31 (2.5 mg kg-1), or saline (control) at the beginning of reperfusion. The difference in blood $\mathrm{H}_{2} \mathrm{O}_{2}$ or $\mathrm{NO}$ between the femoral vein of ischemic and sham limbs in each animal was continuously measured to assess the effects of the drugs.

Key results: In the myocardial I/R model, MitoQ and SS-31 given upon reperfusion significantly improved cardiac function and reduced infarct size compared to untreated control hearts. In the hindlimb I/R model, elevations in blood $\mathrm{H}_{2} \mathrm{O}_{2}$ levels and reductions in blood $\mathrm{NO}$ both indices of elevated ROS, were significantly attenuated by both MitoQ and SS-31 given upon reperfusion compared to saline treated control animals.

Conclusion and implications: The results indicate that mitochondrial-derived ROS is a major contributor to reperfusion injury and that MitoQ and SS-31 work expeditiously to attenuate ROS in that both agents improve cardiac function and limit infarct size when administered only at the onset of reperfusion. The data suggest that MitoQ or SS-31 would be an effective adjuvant to reduce ischemia reperfusion injury in acute myocardial infarction patients receiving percutaneous coronary intervention, thrombolytic therapy, or undergoing coronary by-pass surgery.

\section{Journal of \\ Cardiobiology}

Patel H, Ondrasik R, Galbreath T, Lopez A, Walker S, Chau W, Woodley J, Lepera M, Metellus D, Pham H, Chen Q, Barsotti R and Young LH*

Department of Bio-Medical Sciences, Philadelphia College of Osteopathic Medicine (PCOM), Philadelphia, USA

*Address for Correspondence

Lindon H. Young, Philadelphia College of Osteopathic Medicine (PCOM), Department of Bio-Medical Sciences, 4170 City Avenue, PA 19131, Philadelphia, USA Phone: 215.871.6832, Fax: 215.871.6869; E-mail: Lindonyo@pcom.edu

Submission: 26 April, 2019

Accepted: 25 June, 2019

Published: 28 June, 2019

Copyright: () 2019 Patel H, et al. This is an open access article distributed under the Creative Commons Attribution License, which permits unrestricted use, distribution, and reproduction in any medium, provided the original work is properly cited.

\section{Introduction}

The incidence of Myocardial Infarction (MI) in the United States is 800,000 annually [2]. Prompt treatment to reestablish the blood flow via percutaneous coronary intervention, thrombolytic therapy, or coronary artery by-pass surgery is critical to limit tissue damage, preserve cardiac function and improve clinical outcomes. Paradoxically, abrupt reperfusion of the ischemic myocardium will cause tissue injury, accounting for up to $50 \%$ of the final size of the infarct [3]. Consequently, over the years, numerous studies have attempted to identify agents that limit this Ischemia/Reperfusion $(\mathrm{I} / \mathrm{R})$ injury. However to date, there are no FDA approved treatments for cardiac I/R injury. Therefore, it is critical to understand the underlying mechanisms to develop effective therapeutic strategies to mitigate $\mathrm{I} / \mathrm{R}$ induced cardiac damage $[4,5]$.

Reestablishing perfusion of coronary blood restores the delivery of essential substrates (i.e., oxygen and fatty acids) required to restore ATP levels and normalize $\mathrm{pH}$, however these substrates are also responsible for I/R injury with the majority of cell death occurring during the initial few minutes of reperfusion [6-8]. During severe ischemia, mitochondrial respiration stops, thereby dissipating the membrane potential across the inner membrane and lowering ATP levels. Additionally, calcium $\left(\mathrm{Ca}^{2+}\right)$ accumulation through reversal of the plasma membrane sodium $\left(\mathrm{Na}^{+}\right) / \mathrm{Ca}^{2+}$ exchanger and $\mathrm{Ca}^{2+}$ leakage through the sarcoplasmic reticulum ryanodine receptor channel (RyR2) favors mitochondrial $\mathrm{Ca}^{2+}$ overload, resulting in arrhythmias and hypercontracture [7,9]. Reperfusion is associated with a shift in mitochondria from the production of ATP to Superoxide (SO), specifically from complexes I and III $[10,11]$. The increase in mitochondrial $\mathrm{Ca}^{2+}$ and Reactive Oxygen Species (ROS) leads to the activation of the Mitochondrial Permeability Transition Pore (MPTP), which dissipates mitochondrial membrane potential and uncouples oxidative phosphorylation, further impairing ATP production [11]. Collectively, these events lead to mitochondrial membrane peroxidation and the release of cytochrome $\mathrm{c}$ through the ruptured outer mitochondrial membrane, stimulating apoptotic and necrotic pathways [12].

Under I/R conditions, the mitochondria are a major source 
Citation: Patel H, Ondrasik R, Galbreath T, Lopez A, Walker S, et al. Mitochondrial-Targeted Antioxidants Given at Reperfusion Protect Cardiac and Hindlimb Muscles against Ischemia/Reperfusion Injury. J Cardiobiol. 2019;6(1): 8.

of ROS as they constitute $1 / 3$ of the heart volume; therefore mitochondrial-targeted antioxidants, such as mitoquinone (MitoQ) and SS-31, should be effective pharmacological agents to limit I/R injury [13-15]. The targeted attenuation of mitochondrial ROS would be beneficial by two mechanisms: 1) inhibition of lipid peroxidation would reduce cellular damage and 2) a reduction in peroxynitrite anion (-ONOO) formation by increasing blood Nitric Oxide (NO) bioavailability when mitochondrial-derived SO combines with $\mathrm{NO}$ [16]. Moreover, attenuating mitochondrial-derived SO would also reduce oxidation of the endothelial NO synthase (eNOS) essential cofactor, tetrahydrobiopterin $\left(\mathrm{BH}_{4}\right)$ to dihydrobiopterin $\left(\mathrm{BH}_{2}\right)$. It is well known that when $\mathrm{BH}_{2}$ is increased during reperfusion, eNOS becomes uncoupled and produces SO instead of NO [17-19]. Thus, reducing mitochondrial-derived ROS would also reduce additional ROS generated by uncoupled eNOS during reperfusion [18,20]. Although both MitoQ and SS-31 concentrate their antioxidant properties within mitochondria, their mechanisms targeting the mitochondria differ. MitoQ is covalently attached to a lipophilic Triphenylphosphonium Cation (TPP), which allows it to easily permeate the phospholipid bilayer and concentrate several hundred-fold in the mitochondria [21]. The mitochondrial respiratory chain reduces MitoQ to its active form, ubiquinol, which has been shown to effectively prevent mitochondrial damage and lipid peroxidation [13,22]. In contrast, Szeto-Schiller (SS) peptides contain an alternating cationic and aromatic amino acid with a basic amino acid, e.g., Arg, Lys, providing two positives charges. This alternating cationic-aromatic structural motif enables SS peptides to permeate the plasma cell membrane despite carrying a $3+$ charge at physiological $\mathrm{pH}[14,15]$. Thus, SS peptides uptake by mitochondria is energy independent, nonsaturable and unaffected by the mitochondrial membrane potential.

Additionally, SS-31 contains a dimethyl tyrosine residue that imparts its antioxidant effect. Either agent should reduce I/R-induced mitochondrial ROS production by buffering ROS production at the inner mitochondrial membrane, thereby inhibiting cytochrome c release, mitochondrial swelling, and mitochondrial membrane peroxidation $[21,23]$.

Previous studies with MitoQ or SS-31 have reported effective cardioprotection when administered as a pretreatment or throughout the entire myocardial I/R protocol $[13,23,24]$. More recently, SS-31 was found to limit the I/R injury when administered during ischemia in a hindlimb I/R model [25]. Since pretreatment is often not an option in the clinical scenario of an acute MI, a mitochondrial-directed antioxidant that is effective when administered exclusively during the reperfusion phase would offer more clinical utility. Thus, the primary goal of this study was to test the efficacy of the mitochondrial-directed antioxidants MitoQ and SS-31 when given only during the onset of reperfusion. The effectiveness of these antioxidants in reducing I/R damage was evaluated in myocardial and hindlimb $\mathrm{I} / \mathrm{R}$ models by measuring post-reperfused cardiac function and infarct size, and blood $\mathrm{NO} / \mathrm{H}_{2} \mathrm{O}_{2}$ levels, respectively.

\section{Methods}

\section{Experimental animals and ethical statement}

All animal procedures complied with the legal and ethical guidelines established by the Institutional Animal Care and Use
Committee at PCOM for care and use of animals. Male SpragueDawley (SD) rats 275-325 g (8-10 weeks) (Charles River, Springfield, MA) were housed in polypropylene cages (2 rats in each) lined with wood shavings and provided free access to food and water until the day of the experiment.

\section{Isolated rat heart preparation}

Male SD rats (275-325 g) were anesthetized by Intraperitoneal (i.p.) injection of sodium pentobarbital $\left(60 \mathrm{mg} \mathrm{kg}^{-1}\right)$ containing sodium heparin $(1000 \mathrm{U})$ for anticoagulation. After opening the peritoneum, blood $(8 \mathrm{ml})$ was drawn from the abdominal aorta into a $10 \mathrm{ml}$ syringe that contained $1 \mathrm{ml}$ of citrate phosphate buffer in order to isolate plasma. The heart was isolated from the rat and retroperfused with a modified Krebs' buffer through the aorta using the Langendorff protocol [26]. As described in previous studies, the aorta was cannulated with a modified 18 gauge syringe needle and secured using 0 grade silk thread. The preparation was lowered into a temperature-controlled reservoir that was filled with Krebs' buffer maintained at $\mathrm{pH}$ of 7.35-7.45 by aerating continuously with $95 \% \mathrm{O}_{2}-5 \% \mathrm{CO}_{2}$ at $37{ }^{\circ} \mathrm{C}$. A constant pressure of $80 \mathrm{mmHg}$ was maintained throughout the experiment. The Krebs' buffer contained: $25 \mathrm{mM} \mathrm{NaHCO}, 17 \mathrm{mM}$ dextrose, $5.9 \mathrm{mM} \mathrm{KCl}, 120 \mathrm{mM} \mathrm{NaCl}, 0.5$ mM EDTA, $2.5 \mathrm{mM} \mathrm{CaCl}_{2}$, and $1.2 \mathrm{mM} \mathrm{MgCl}_{2}$. Cardiac function parameters: Left Ventricular End-Systolic Pressure (LVESP), Left Ventricular End-Diastolic Pressure (LVEDP), maximal rate of left ventricular developed pressure generation $\left(+\mathrm{dP} / \mathrm{dt}_{\max }\right)$ and decline $\left(-\mathrm{dP} / \mathrm{dt}_{\min }\right)$, heart rate, and coronary flow were recorded throughout the entire experiment using a pressure transducer (SPR-524, Millar Instruments, Inc., Houston, TX) positioned in the left ventricle and an in-line flow meter (T106, Transonic Systems, Inc., Ithaca, NY). Left Ventricular Developed Pressure (LVDP) was calculated continuously by subtracting LVEDP from LVESP. Data were continuously recorded and stored using a Powerlab station acquisition system (PowerLab/8Sp, AD Instruments, Grand Junction, CO) [18,19,27].

\section{Myocardial I/R procedure}

After the baseline period (15 min), global ischemia was induced for $30 \mathrm{~min}$ followed by a $45 \mathrm{~min}$ reperfusion period. Hearts were randomized to receive either $5 \mathrm{ml}$ of plasma (control), plasma containing SS-31 $(10 \mu \mathrm{M}, 25 \mu \mathrm{M}, 50 \mu \mathrm{M}, 100 \mu \mathrm{M})$, or Mito Q $(1 \mu \mathrm{M}$, $10 \mu \mathrm{M}, 20 \mu \mathrm{M})$. Stock concentrations of SS-31 (50 mM) and MitoQ $(20 \mathrm{mM})$ were prepared in deionized $\mathrm{H}_{2} \mathrm{O}$ and further diluted in plasma. Drug + plasma or plasma alone was administered to the heart during the first $5 \mathrm{~min}$ of reperfusion at a rate of $1 \mathrm{ml} / \mathrm{min}$ using an infusion pump. At the end of the experiment, the heart was crosssectioned into $2 \mathrm{~mm}$ sections from apex to base. The heart crosssections were subjected to $1 \%$ Triphenyltetrazolium Chloride (TTC) staining for $15 \mathrm{~min}$ at $37^{\circ} \mathrm{C}$ to determine infarct size as previously described $[20,27,28]$.

\section{Hindlimb I/R procedure}

Male SD rats were anesthetized with an i.p. injection of sodium pentobarbital (60 mg kg-1 induction dose, $30 \mathrm{mg} \mathrm{kg}^{-1}$ maintenance doses as needed). The hindlimb was dissected to expose the femoral veins and arteries bilaterally. Both femoral veins were cannulated with a 24-gauge catheter housing a calibrated $\mathrm{H}_{2} \mathrm{O}_{2}$ or $\mathrm{NO}$ microsensor (100 $\mu \mathrm{m}$ diameter, World Precision Instruments (WPI) Inc., Sarasota, 


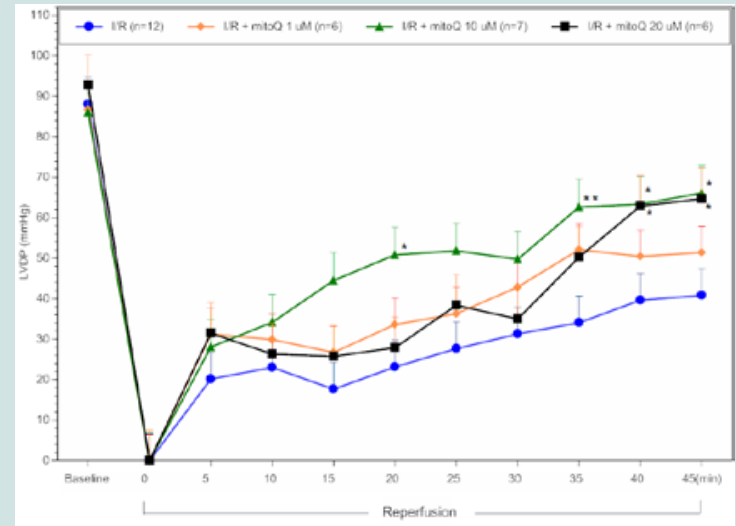

Figure 1: LVDP timecourse for MitoQ I/R hearts. All data were analyzed using ANOVA with the Student-Newman-Keuls test. Final LVDP (45 min reperfusion) was significantly improved in MitoQ I/R treated hearts (10 \&20 $\mu \mathrm{M})$ compared to control I/R hearts $\left({ }^{*} \mathrm{p}<0.05 ;{ }^{* *} \mathrm{p}<0.01\right.$ compared to control $\mathrm{I} / \mathrm{R})$

FL) that were inserted into the catheters bilaterally. The microsensors were connected to a free radical analyzer (Apollo 4000, WPI, Inc., Sarasota, FL). This arrangement allowed for real-time measurements of $\mathrm{H}_{2} \mathrm{O}_{2}$ or $\mathrm{NO}$ release as previously described $[18,20,29]$. One limb was subjected to $\mathrm{I} / \mathrm{R}$ via clamping of the femoral artery and vein for 30 min followed by reperfusion for $45 \mathrm{~min}$. Meanwhile, the other limb was used as a non-ischemic sham control in the same rat. The rats were randomized to receive either MitoQ $\left(1.8 \mathrm{mg} \mathrm{kg}^{-1}\right)$, SS-31 $\left(2.5 \mathrm{mg} \mathrm{kg}^{-1}\right)$, or saline (non-drug control) via i.v. bolus into the left jugular vein at the beginning of reperfusion. These doses were estimated to result in blood levels of 10 and $50 \mu \mathrm{M}$, respectively. These concentrations were chosen because they resulted in significant cardioprotective effects in the isolated rat heart experiments. Blood $\mathrm{H}_{2} \mathrm{O}_{2}$ or $\mathrm{NO}$ release was continuously monitored and values were recorded at 5 min intervals. Values measured from the I/R limb were subtracted from the sham limb to determine the net relative difference in $\mathrm{H}_{2} \mathrm{O}_{2}$ or NO release over the course of the experiment. At the conclusion of the experiment, while still under anesthesia, the rats were sacrificed by opening the thoracic cavity and removing the heart.

\section{Materials}

MitoQ (MW=579 $\mathrm{g} \mathrm{mol}^{-1}$ ) used in this study was completed with cyclodextrin $\left(\mathrm{MW}=1135 \mathrm{~g} \mathrm{~mol}^{-1}\right)$ to improve water solubility (total MW=1714 $\mathrm{g} \mathrm{mol}^{-1}$, Vosun Shop, Suzhou China). SS-31 peptide: D-Arg-Dmt-Lys-Phe-Amide (MW=640 $\mathrm{g} \mathrm{mol}^{-1}$ ) was synthesized by Genemed Synthesis, Inc., San Antonio, TX.TTC was purchased from Sigma-Aldrich, St. Louis, MO. All other reagents were purchased from Fisher Scientific, Fair Lawn, NJ.

\section{Statistical analysis}

All data in the text and figures are presented as means \pm standard error of the mean (S.E.M.). In the myocardial I/R studies, the data were analyzed post hoc by analysis of variance (ANOVA) with the Student-Newman-Keuls test, while a Student's t-test was used to analyze the data from the hindlimb I/R experiments. In all cases, a $\mathrm{p}$ value $<0.05$ was considered to be statistically significant.

\section{Results}

Effects of MitoQ and SS-31 on cardiac function and infarct size
We examined the effects of MitoQ and SS-31 by measuring the following cardiac function parameters throughout the experiment (Table 1): LVEDP, LVESP, heart rate, coronary flow, $+\mathrm{dP} / \mathrm{dt}_{\max }$, and $-\mathrm{dP} / \mathrm{dt}_{\min }$. Sham hearts were not subjected to ischemia and maintained near normal cardiac function parameters throughout the 90 min experimental period. There were no significant differences between the initial and final values in all cardiac function indices and minimal cell death (infarct size was less than 0.05\%; data not shown). Also, there were no significant differences in baseline (preischemia) cardiac function values among all experimental groups. I/R hearts treated by infusion of MitoQ $(10$ or $20 \mu \mathrm{M})$ and SS-31 $(50 \mu \mathrm{M})$ during the first $5 \mathrm{~min}$ of reperfusion exhibited significantly improved postreperfused cardiac contractile function and reduced infarct size compared to controls. Post-reperfused LVDP at the end of reperfusion recovered to $59 \pm 12 \%(1 \mu \mathrm{M} ; \mathrm{p}>0.05, \mathrm{n}=6), 77 \pm 6 \%$ $(10 \mu \mathrm{M} ; \mathrm{p}<0.05, \mathrm{n}=7), 70 \pm 10 \%(20 \mu \mathrm{M} ; \mathrm{p}<0.05, \mathrm{n}=6)$ in I/R MitoQ treated hearts when compared to control I/R hearts that recovered to $46 \pm 6 \%(n=12)$ of initial baseline values. In SS-31 treated hearts, post-reperfused LVDP at the end of reperfusion recovered to $38 \pm 3 \%$ $(10 \mu \mathrm{M} ; \mathrm{p}>0.05, \mathrm{n}=6), 48 \pm 7 \%(25 \mu \mathrm{M} ; \mathrm{p}>0.05, \mathrm{n}=6), 80 \pm 6 \%(50 \mu \mathrm{M}$; $\mathrm{p}<0.01, \mathrm{n}=6)$, and $62 \pm 8 \%(100 \mu \mathrm{M} ; \mathrm{p}>0.05, \mathrm{n}=6)$ compared to control $\mathrm{I} / \mathrm{R}$ hearts that recovered to $46 \pm 6 \%(\mathrm{n}=12)$ of baseline values. As shown in (Figure 1), MitoQ infusion $(10 \mu \mathrm{M}$ and $20 \mu \mathrm{M})$ resulted in a significant improvement in post-reperfused LVDP from 35 to 45 min compared to controls. In contrast, similar concentrations of SS31 (10 and $25 \mu \mathrm{M}$, Figure 2), showed no significant improvement in cardiac function. Improvement in post-reperfused cardiac function was observed when the SS-31 concentration was further increased to $50 \mu \mathrm{M}$. As illustrated in (Figure 2), at this concentration of SS-31(50 $\mu \mathrm{M})$, a significant improvement in LVDP was observed at $15 \mathrm{~min}$ post-reperfusion, and sustained throughout the reperfusion period (45 min) compared to control I/R hearts $(\mathrm{p}<0.01)$. However, similar to MitoQ, the improvement in post-reperfused LVDP was associated with a significant increase in final post-reperfused LVESP $(131 \pm 11$ $\mathrm{mmHg}$ [MitoQ $20 \mu \mathrm{M}$ ]; $127 \pm 5 \mathrm{mmHg}$ [SS-31 $50 \mu \mathrm{M}$ ] vs. $102 \pm 6$ $\mathrm{mmHg}$ [Control]; $\mathrm{p}<0.05$ ) and not a decrease in LVEDP.

Unlike cardiac function, all doses of MitoQ (except $1 \mu \mathrm{M}$ ) and SS31 used in these studies significantly improved (reduced) infarct size

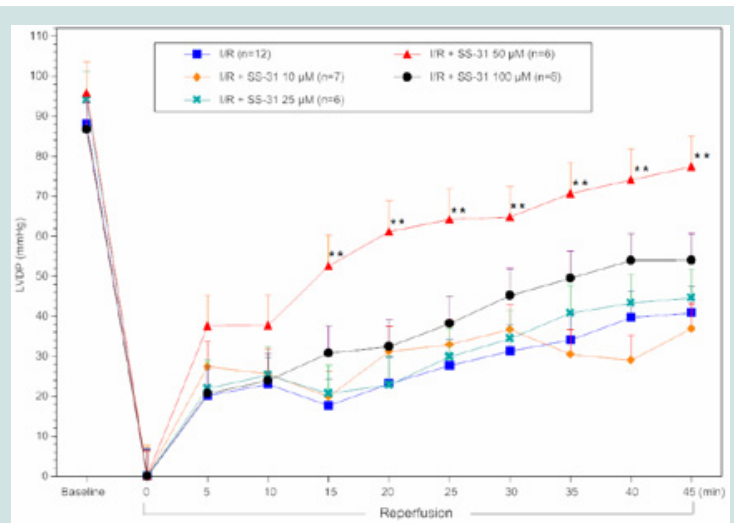

Figure 2: LVDP timecourse for SS-31 I/R hearts. All data were analyzed using ANOVA with the Student-Newman-Keuls test. Final LVDP (45min reperfusion) was significantly improved in SS-31 treated $(50 \mu \mathrm{M})$ hearts compared to control I/R hearts $\left({ }^{* *} p<0.01\right.$ compared to control I/R). 
Citation: Patel H, Ondrasik R, Galbreath T, Lopez A, Walker S, et al. Mitochondrial-Targeted Antioxidants Given at Reperfusion Protect Cardiac and Hindlimb Muscles against Ischemia/Reperfusion Injury. J Cardiobiol. 2019;6(1): 8.

Table 1: Cardiac function initial (baseline) and final values for control I/R, I/R + MitoQ (1-20 $\mu \mathrm{M}), \mathrm{I} / \mathrm{R}+\mathrm{SS} 31(10-50 \mu \mathrm{M})$ treated hearts and infarct size. LVESP, left ventricular end systolic pressure; LVEDP, left ventricular end diastolic pressure; LVDP, left ventricular developed pressure; maximal rate of LV pressure generation

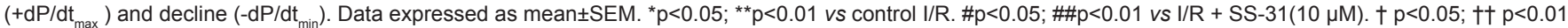
$v s \mathrm{I} / \mathrm{R}+\mathrm{SS}-31(25 \mu \mathrm{M}) \omega \mathrm{p}<0.05 ; \omega \omega \mathrm{p}<0.01$ vs. SS-31 $(100 \mu \mathrm{M}) \cdot \psi \mathrm{p}<0.05 v s \mathrm{I} / \mathrm{R}+\mathrm{MitoQ}(1 \mu \mathrm{M}) . \psi \psi \mathrm{p}<0.01 v s \mathrm{I} / \mathrm{R}+\mathrm{MitoQ}(1 \mu \mathrm{M})$.

\begin{tabular}{|c|c|c|c|c|c|c|c|c|}
\hline & $\begin{array}{c}\text { I/R Control } \\
\text { (plasma) } \\
(n=12)\end{array}$ & $\begin{array}{c}\mathrm{I} / \mathrm{R}+\mathrm{MitoQ} 1 \\
\mu \mathrm{M}(\mathrm{n}=6)\end{array}$ & $\begin{array}{c}\mathrm{I} / \mathrm{R}+\mathrm{MitoQ} 10 \\
\mu \mathrm{M}(\mathrm{n}=7)\end{array}$ & $\begin{array}{c}\text { I/R + MitoQ } 20 \\
\mu M(n=6)\end{array}$ & $\begin{array}{c}\mathrm{I} / \mathrm{R}+\mathrm{SS}-3110 \\
\mu \mathrm{M}(\mathrm{n}=7)\end{array}$ & $\begin{array}{c}\mathrm{I} / \mathrm{R}+\mathrm{SS}-3125 \\
\mu M(n=6)\end{array}$ & $\begin{array}{c}\mathrm{I} / \mathrm{R}+\mathrm{SS}-3150 \mu \mathrm{M} \\
(\mathrm{n}=6)\end{array}$ & $\begin{array}{c}I / R+S S-31100 \\
\mu M(n=6)\end{array}$ \\
\hline Initial LVESP (mmHg) & $99 \pm 4$ & $99 \pm 4$ & $96 \pm 2$ & $104 \pm 3$ & $94 \pm 2$ & $104 \pm 3$ & $107 \pm 3$ & $96 \pm 2$ \\
\hline Initial LVEDP (mmHg) & $11 \pm 1$ & $12 \pm 1$ & $10 \pm 1$ & $11 \pm 1$ & $7 \pm 1$ & $10 \pm 1$ & $11 \pm 1$ & $9 \pm 0.3$ \\
\hline Initial LVDP $(\mathrm{mmHg})$ & $88 \pm 4$ & $87 \pm 4$ & $86 \pm 2$ & $93 \pm 2$ & $87 \pm 2$ & $94 \pm 3$ & $96 \pm 3$ & $87 \pm 2$ \\
\hline Final LVESP $(\mathrm{mmHg})$ & $102 \pm 6$ & $115 \pm 6$ & $115 \pm 8$ & $131 \pm 11^{*}$ & $85 \pm 6$ & $103 \pm 5$ & $127 \pm 5$ *\#\#† & $103 \pm 10$ \\
\hline Final LVEDP $(\mathrm{mmHg})$ & $61 \pm 5$ & $63 \pm 5$ & $49 \pm 6 \psi$ & $66 \pm 7$ & $52 \pm 6$ & $59 \pm 3$ & $50 \pm 7$ & $49 \pm 6$ \\
\hline Final LVDP $(\mathrm{mmHg})$ & $41 \pm 5$ & $51 \pm 10$ & $66 \pm 5^{*}$ & $65 \pm 9^{*}$ & $33 \pm 5$ & $45 \pm 7$ & $77 \pm 5^{\star \star \# \# † † ~ \omega \omega ~}$ & $54 \pm 7 \#$ \\
\hline Initial+dP/dt ${ }_{\text {max }}(\mathrm{mmHg} / \mathrm{s})$ & $2349 \pm 78$ & $2218 \pm 72$ & $2226 \pm 60$ & $2342 \pm 40$ & $2211 \pm 25$ & $2270 \pm 66$ & $2216 \pm 48$ & $2182 \pm 41$ \\
\hline Final $+\mathrm{dP} / \mathrm{dt}_{\max }(\mathrm{mmHg} / \mathrm{s})$ & $882 \pm 113$ & $912 \pm 193$ & $1381 \pm 110^{*} \Psi$ & $1294 \pm 148^{*} \Psi$ & $674 \pm 120$ & $970 \pm 98$ & $1565 \pm 162 * \# \#+† \omega \omega$ & $1069 \pm 76 \#$ \\
\hline Initial-dP/dt ${ }_{\min }(\mathrm{mmHg} / \mathrm{s})$ & $-1570 \pm 77$ & $-1436 \pm 50$ & $-1420 \pm 36$ & $-1486 \pm 50$ & $-1448 \pm 37$ & $-1629 \pm 40$ & $-1540 \pm 70$ & $-1429 \pm 59$ \\
\hline Final-dP/dt ${ }_{\text {min }}(\mathrm{mmHg} / \mathrm{s})$ & $-769 \pm 56$ & $-858 \pm 157$ & $-1025 \pm 115$ & $-948 \pm 187$ & $-526 \pm 80$ & $-704 \pm 101$ & $-1256 \pm 120^{* *} \# \#+†$ & $-886 \pm 149 \#$ \\
\hline $\begin{array}{c}\text { Final Coronary Flow (mL/ } \\
\text { min) }\end{array}$ & $8 \pm 0.4$ & $9 \pm 1$ & $12 \pm 3$ & $8 \pm 1$ & $8 \pm 1$ & $10 \pm 1$ & $11 \pm 1$ & $9 \pm 1$ \\
\hline Initial Heart Rate (BPM) & $278 \pm 16$ & $251 \pm 14$ & $273 \pm 12$ & $277 \pm 14$ & $266 \pm 5$ & $282 \pm 20$ & $261 \pm 7$ & $300 \pm 9$ \\
\hline Final Heart Rate (BPM) & $250 \pm 14$ & $240 \pm 20$ & $238 \pm 23$ & $243 \pm 21$ & $232 \pm 8$ & $257 \pm 15$ & $265 \pm 16$ & $262 \pm 12$ \\
\hline Infarct Size (\%) & $45 \pm 2$ & $54 \pm 6$ & $27 \pm 3^{* *} \psi \psi$ & $23 \pm 2 * * \psi \psi \psi$ & $36 \pm 2$ * & $25 \pm 4$ **\#\# & $19 \pm 2 * \# \#$ & $21 \pm 2 * \# \#$ \\
\hline
\end{tabular}

when compared to controls as shown in (Table 1). The concentrations of MitoQ and SS-31 that showed the greatest effect, reduced infarct size to $23 \pm 2 \%(20 \mu \mathrm{M}, \mathrm{p}<0.01, \mathrm{n}=6)$ and $19 \pm 2 \%(50 \mu \mathrm{M}, \mathrm{p}<0.01$, $\mathrm{n}=6$ ), respectively, compared to the control I/R group, which showed an infarct size of $45 \pm 2 \%$. Thelowest infarct sizes also correlated with robust and significant recovery in post-reperfused LVDP at $45 \mathrm{~min}$ observed in MitoQ treated $[(20 \mu \mathrm{M}) ; \mathrm{p}<0.05, \mathrm{n}=6]$; SS-31 treated [(50 $\mu \mathrm{M}) ; \mathrm{p}<0.01, \mathrm{n}=6])$ compared to control I/R hearts $(\mathrm{n}=12)$ (Table 1$)$.

\section{Effects of MitoQ and SS-31 on ROS and NO bioavailability in blood}

We compared the effects of MitoQ and SS-31 in reducing blood ROS as measured as a decrease in $\mathrm{H}_{2} \mathrm{O}_{2}$ and an increase in bioavailability of $\mathrm{NO}$ in a rat hindlimb $\mathrm{I} / \mathrm{R}$ model. Our data depicts the relative difference between the I/R limb and the non-ischemic or sham limb in animals treated with drug or saline (control). As shown in (Figure 3), rats treated with a bolus of MitoQ $\left(1.8 \mathrm{mg} \mathrm{kg}^{-1} \sim 10 \mu \mathrm{M}\right.$ in blood) at the onset of reperfusion had a significant reduction in blood levels of $\mathrm{H}_{2} \mathrm{O}_{2}$ beginning at $10 \mathrm{~min}$ after infusion and maintained this effect throughout the reperfusion period compared to controls receiving saline. Similarly, rats treated with SS-31 $\left(2.5 \mathrm{mg} \mathrm{kg}^{-1} \sim 50\right.$ $\mu \mathrm{M}$ in blood) at reperfusion had significantly decreased $\mathrm{H}_{2} \mathrm{O}_{2}$ levels compared to the control group. This effect was observed at $15 \mathrm{~min}$ and maintained throughout the reperfusion period (Figure 4). In contrast to blood $\mathrm{H}_{2} \mathrm{O}_{2}$ levels, $\mathrm{NO}$ bioavailability was significantly higher beginning at $20 \mathrm{~min}$ post reperfusion in rats treated with MitoQ (1.8 $\mathrm{mg} \mathrm{kg}^{-1}, \sim 10 \mu \mathrm{M}$ in blood) or SS-31 (2.5 mg kg-1,$~ 50 \mu \mathrm{M}$ in blood) compared to saline controls (Figure 5), and this effect was maintained throughout the reperfusion period (Figure 6).

\section{Discussion and Conclusion}

The major findings of this study are: 1) Both MitoQ and SS-
31 treatment initiated at the onset of reperfusion restored postreperfused cardiac function and reduced infarct size compared to controls in isolated rat hearts subjected to $30 \mathrm{~min}$ of ischemia and 45 min of reperfusion; 2) MitoQ and SS-31 significantly decreased blood $\mathrm{H}_{2} \mathrm{O}_{2}$ and increased blood NO bioavailability following hindlimb I/R injury compared to saline controls. The increased NO bioavailability effect was most likely due to a reduction in NO scavenging by SO. Together, these results indicate that mitochondrial-targeted antioxidant agents are effective in reducing I/R injury when given only at reperfusion by reducing mitochondrial ROS production and improving NO bioavailability.

\section{Myocardial I/R Model}

The ex vivo myocardial I/R model has proven to be reliable to screen potential drug candidates to reduce reperfusion-induced cellular injury $[20,26,27,30,31]$. Drug candidates that have shown reduction in infarct size and improved post-reperfused function in ex vivo studies are then tested using in vivo myocardial I/R models [32-34]. Previous studies have shown that both MitoQ and SS-31 were able to reduce infarct size when given as a pretreatment or preconditioning, i.e., prior to the initiation of a prolonged ischemia period $[13,24,35]$. The novelty of our study is that we tested both of these mitochondrial-directed antioxidants when given only briefly, during the first $5 \mathrm{~min}$ of reperfusion following $30 \mathrm{~min}$ of ischemia. This is an important test to evaluate the therapeutic benefit of such compounds since pretreatment is often not an option prior to percutaneous coronary intervention. In addition, many potential drug candidates that have worked when given as a pretreatment in preclinical myocardial I/R studies, e.g., cyclosporine and exenatide, have not translated to the clinical setting of myocardial infarction 


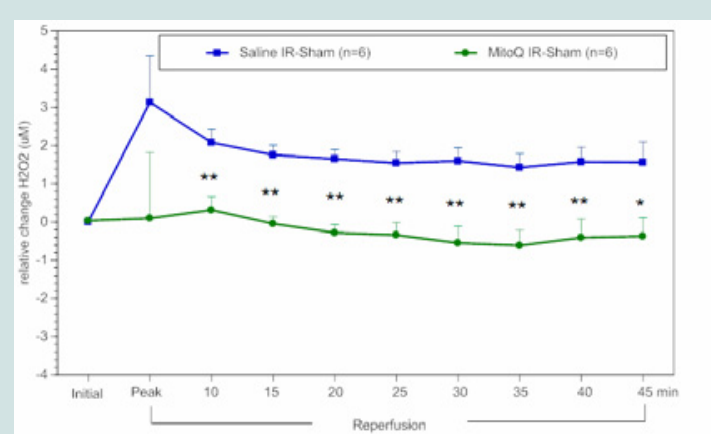

Figure 3: Timecourse of the relative difference in $\mathrm{H}_{2} \mathrm{O}_{2}$ release of $\mathrm{I} / \mathrm{R}$ limbs compared to the other sham limbs (I/R-sham) during reperfusion in MitoQ (1.8 $\mathrm{mg} \mathrm{kg-1)}$ or saline treated animals. The blue squares represent the relative difference in $\mathrm{H}_{2} \mathrm{O}_{2}$ release between the sham limb and I/R limb in the saline treated (control) group. The green circles represent the relative difference in $\mathrm{H}_{2} \mathrm{O}_{2}$ release between the sham limb and I/R limb in the MitoQ treated group. All data were analyzed using Student's t-test $\left({ }^{*} p<0.05\right.$; ${ }^{* *} p<0.01$ compared to saline control).

[36,37]. In part, this may be due to the inefficient targeting to key sources of ROS or other necrotic pathways active at the beginning of reperfusion or limited accessibility of the therapeutic agent to its target pathway. It is more likely that agents that are effective in preconditioning the myocardium fail as postconditioning agents because cardioprotective pathways prior to the ischemia are different from cardioprotective pathways after the ischemic insult $[27,30]$. In general, the effectiveness of these mitochondrial-directed therapeutic agents, specifically when given upon the onset of reperfusion and for only a brief time period ( $5 \mathrm{~min}$ ), strongly support the hypothesis that the mitochondria play a critical role in mediating reperfusioninduced cell death in the early minutes following the reestablishment of flow.

\section{A. Cardiac function}

Interestingly in this study, MitoQ $(10-20 \mu \mathrm{M})$ and SS-31 $(50 \mu \mathrm{M})$ were found to be the most effective doses in restoring post-reperfused cardiac function. Specifically, the component of LVDP that seems to be responsible for this improved function was the significant elevation in LVESP (i.e., MitoQ $20 \mu \mathrm{M}$ and SS-31 $50 \mu \mathrm{M}$ ) compared to controls. This may be related to improving mitochondrial function, which in turn would lead to greater ATP production and an improvement in cardiac contractility during reperfusion [3].

The unique finding in our study is that both MitoQ and SS-31 given only during the first $5 \mathrm{~min}$ of reperfusion was sufficient to restore cardiac function, thus, need not be given throughout reperfusion or as pretreatment (prior to ischemia) as reported in previous studies $[13,24,35]$. Mechanistically, this may be related to inhibiting mitochondrial ROS during reperfusion and reducing cardiolipin peroxidation, thus maintaining the electron transport chain function, preserving mitochondrial membrane potential and ATP synthesis, and maintaining normal $\mathrm{Ca}^{2+}$ handling by the cardiomyocyte leading to enhancement of post-reperfused cardiac function [7]. Previous reports on the cardioprotective effect of MitoQ on I/R injury in isolated rat heart have described using this agent prior to prolonged ischemia (preconditioning) to limit mitochondrial damage during the ischemic period, which in turn would also presumably preserve mitochondrial function at reperfusion $[13,23,24]$. By contrast, the results from our study implicate that inhibiting mitochondrialderived ROS production briefly during the onset of reperfusion is sufficient to restore post-reperfused cardiac function and attenuate infarct size. In general, the present findings further support the premise that MitoQ inhibits lethal ROS induced I/R injury [4,24]. A previous study using SS-31 on isolated guinea pig hearts subjected to I/R (30 min ischemia and $90 \mathrm{~min}$ reperfusion) reported that SS31 infused during baseline (prior to ischemia) and throughout the reperfusion period or throughout the reperfusion period only (no baseline infusion) significantly improved post-reperfused cardiac contractile function [35]. By contrast, we found in the present study that SS-31 or MitoQ infused during only the first $5 \mathrm{~min}$ of a $45 \mathrm{~min}$ reperfusion period was sufficient to significantly reduce I/R injury, most likely via a reduction in mitochondrial produced ROS.

As shown in (Figure 2), while treatment with a $10 \mu \mathrm{M}$ concentration of SS-31 significantly reduced infarct size, it did not restore cardiac function, suggesting that the viable myocardium was stunned and unable to fully recover post-reperfusion cardiac function within $45 \mathrm{~min}$ of reperfusion. Stunning is a phenomena where the heart does not recover function immediately following reperfusion that is not accounted for by cardiomyocyte death or reduced blood flow and may take several days to weeks before returning to normal. Consistent with this observation, another study of SS-31, also known as Bendavia, reported that the compound reduced infarct size in two of three myocardial I/R models: (in vivo sheep and ex vivo guinea pig hearts showed a significant reduction of infarct size, and in vivo rabbit hearts showed a trend of reduced infarct size). However, cardiac function (i.e., LVDP of guinea pig hearts ex vivo and ejection fraction in sheep and rabbit hearts in vivo) did not recover in any of the three models [38]. Collectively, the data from the current study and others suggest that post ischemic administration of SS-31 is effective in reducing infarct size over a relatively broad dose range, but myocardial stunning may persist since cardiac function did not recover over the reperfusion period tested (45 min). Interestingly, studies on isolated mouse liver mitochondria [23], showed that over the same concentration range used in the present study, SS-31 concentration dependently inhibited ROS in isolated mitochondria

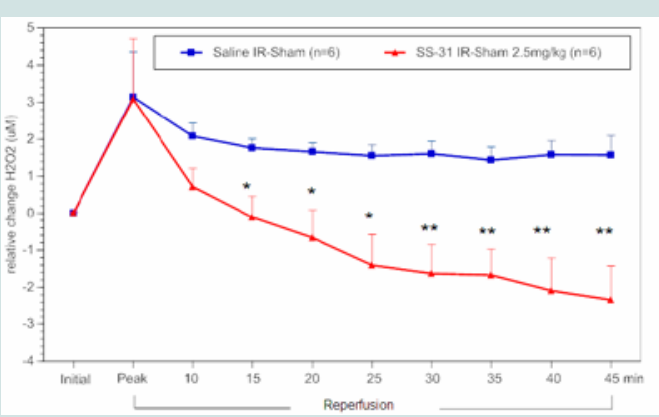

Figure 4: Time course of the relative difference in $\mathrm{H}_{2} \mathrm{O}_{2}$ release of $\mathrm{I} / \mathrm{R}$ limbs compared to their sham limbs (I/R-sham) during reperfusion in SS-31(2.5 $\mathrm{mg} \mathrm{kg-1)}$ or saline treated animals. The blue squares represent the relative difference in $\mathrm{H}_{2} \mathrm{O}_{2}$ release between the sham limb and I/R limb in the saline treated (control) group. The red triangles represent the relative difference in $\mathrm{H}_{2} \mathrm{O}_{2}$ release between the sham limb and I/R limb in the SS-31 treated group. All data were analyzed using Student's t-test $\left({ }^{*} p<0.05 ;{ }^{* *} p<0.01\right.$ compared to saline control). 
Citation: Patel H, Ondrasik R, Galbreath T, Lopez A, Walker S, et al. Mitochondrial-Targeted Antioxidants Given at Reperfusion Protect Cardiac and Hindlimb Muscles against Ischemia/Reperfusion Injury. J Cardiobiol. 2019;6(1): 8.

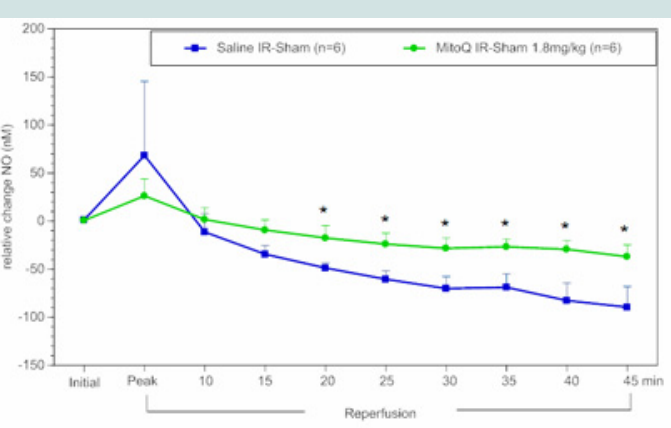

Figure 5: Time course of the relative difference in NO release of $I / R$ limbs compared to their sham limbs (I/R-sham) during reperfusion in MitoQ (1.8 $\mathrm{mg} \mathrm{kg}-1$ ) or saline treated animals. The blue squares represent the relative difference in NO release between the sham limb and I/R limb in the saline treated (control) group. The green circles represent the relative difference in $\mathrm{NO}$ release between the sham limb and I/R limb in the MitoQ treated group. All data were analyzed using Student's t-test $\left({ }^{*} p<0.05\right.$ compared to saline control).

induced by 3-nitropropionic acid, a potent complex II toxin. These results, along with the hindlimb I/R studies described earlier suggest that inhibiting mitochondrial-derived ROS underlies the improved post-reperfused cardiac function and reduced infarct size found with SS-31 and MitoQ treatment. Collectively, the data indicate that MitoQ significantly improved final post-reperfused LVDP compared to controls, and reached a maximum effect between 10 to $20 \mu \mathrm{M}$ with this mitochondrial anti-oxidant agent (Table 1). While SS-31 also exhibited a concentration dependent effect to improve cardiac function as indicated by the increase in final LVDP (i.e., 10 $\mu \mathrm{M}[33 \pm 5 \mathrm{mmHg}] ; 25 \mu \mathrm{M}[45 \pm 7 \mathrm{mmHg}]$ vs. $50 \mu \mathrm{M}[77 \pm 5 \mathrm{mmHg}])$, the $100 \mu \mathrm{M}[54 \pm 7 \mathrm{mmHg}])$ exhibited a decrease in post-reperfused cardiac function compared to hearts treated with $50 \mu \mathrm{M}$. These latter results suggest that SS-31 at $100 \mu \mathrm{M}$ may induce post-reperfused cardiac stunning. A similar effect was also reported in ex vivo guinea pig hearts and in vivo rabbit and sheep hearts in the Bendavia study [38].

\section{B. Infarct size}

In a previous study, Adlam et al. provided MitoQ $(500 \mu \mathrm{M})$ in drinking water as pretreatment for two weeks and reported a significant reduction in lactate dehydrogenase activity in coronary effluent of isolated hearts [13], in cytochrome $\mathrm{c}$ release from mitochondria into the cytosol and in caspase 3 upregulation, all indicating a reduction in cardiac tissue damage. By contrast, in this study, both MitoQ (10 and $20 \mu \mathrm{M})$ and SS-31 (10-100 $\mu \mathrm{M})$ reduced infarct size when compared to untreated controls when given during the initial $5 \mathrm{~min}$ of reperfusion (Table 1). This finding is clinically relevant since it indicates that these mitochondrial-directed compounds could be effective after ischemic injury has occurred and thus, could be given in a clinical setting to limit I/R injury, during percutaneous coronary intervention, thrombolytic treatment, or by-pass surgery. Likewise, Szeto reported that SS-31 is effective in reducing infarct size in isolated hearts subjected to global ischemia when given throughout the $90 \mathrm{~min}$ reperfusion period [14]. In contrast, in the present study we found SS-31 was effective in both improving cardiac function and reducing infarct size when given during only the first $5 \mathrm{~min}$ of reperfusion (Table 1). Collectively, the data show that MitoQ exhibited a concentration dependent effect in the reduction of infarct size $(1 \mu \mathrm{M}[54 \pm 6]$ vs. $10 \mu \mathrm{M}[27 \pm 3]$ and 20 $\mu \mathrm{M}[23 \pm 2])$ that paralleled the improvement in post-reperfused LVDP. By contrast, SS-31 treated hearts showed a concentration-dependent effect in reducing infarct size, and this effect was maximally obtained in the $50 \mu \mathrm{M}[19 \pm 2 \%]$ to $100 \mu \mathrm{M}[21 \pm 2 \%]$ concentration tested. The effects of SS-31 in this study are consistent with the findings in the Bendavia study (38) and suggest SS-31 can still exert myocardial tissue salvaging effects (i.e., reduced cell death) without an accompanying increase in post-reperfused cardiac function (e.g., LVDP) since only the $50 \mu \mathrm{M}$ exhibited significant improvement in post-reperfused cardiac function compared to controls. Moreover, the infarct sparing effects of SS-31 in concentrations that did not result in significantly improved post-reperfused cardiac function compared to controls during the $45 \mathrm{~min}$ reperfusion period suggest that the heart may be experiencing stunning and could potentially recover cardiac function as stunning subsided.

Collectively, the effects of MitoQ $(10 \& 20 \mu \mathrm{M})$ and SS-31 (all concentrations) on infarct size are similar despite differences in their mode of entry into the mitochondria. The positive charge on the MitoQ molecule is essential for entry into the negatively-charged mitochondrial membrane potential $[13,21]$. By contrast, SS-31 can enter the mitochondria independently of the mitochondrial membrane potential [14]. During global ischemia, mitochondrial membrane potential likely dissipated. Nevertheless, the results of this study would suggest that both of these mitochondrial-directed antioxidants are able to rapidly enter the mitochondria within the first 5 minutes of reperfusion to preserve mitochondrial membrane potential and decreased mitochondrial ROS production compared to untreated control hearts.

\section{Hindlimb I/R model}

We used the hindlimb I/R animal model to measure changes in blood $\mathrm{H}_{2} \mathrm{O}_{2}$ and NO in real-time in rats treated with MitoQ and SS31 at reperfusion. We chose this model due to the ability to measure blood $\mathrm{H}_{2} \mathrm{O}_{2}$ as an index of blood ROS levels that may occur in the coronary circulation during reperfusion injury, thought to result from both endothelial and cardiomyocyte damage. Moreover, our previous studies have shown consistently that agents which reduce blood $\mathrm{H}_{2} \mathrm{O}_{2}$

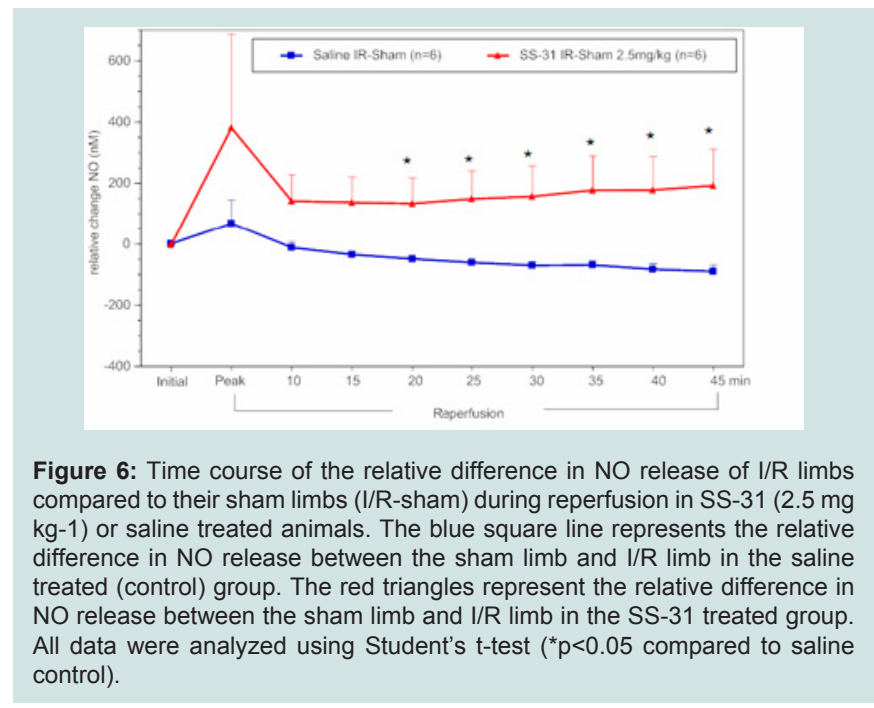


Citation: Patel H, Ondrasik R, Galbreath T, Lopez A, Walker S, et al. Mitochondrial-Targeted Antioxidants Given at Reperfusion Protect Cardiac and Hindlimb Muscles against Ischemia/Reperfusion Injury. J Cardiobiol. 2019;6(1): 8.

and increase blood NO in hindlimb I/R also improve post-reperfused cardiac function and reduce infarct size $[18,19,20,31,39]$.

Inhibition of mitochondrial-derived ROS release would attenuate the quenching of endothelial-derived NO (i.e., less -OONO formation) and thus lead to restoration of post-reperfused cardiac function, reduction of infarct size, and inhibition of inflammatory cytokines released from ROS-damaged tissue $[4,24,40]$. As expected, the bioavailability of blood NO following I/R injury was significantly greater in the groups treated with MitoQ or SS-31 compared to the controls, which only received saline at reperfusion. This is likely related to the decrease in blood $\mathrm{H}_{2} \mathrm{O}_{2}$ in treated animals. Blood $\mathrm{H}_{2} \mathrm{O}_{2}$ serves as an index of $\mathrm{SO}$, given the short half-life of SO. When SO scavenges NO, it produces the harmful -ONOO, which further exacerbates reperfusion-induced ROS tissue damage and leads to a further decrease in the bioavailability of NO [16]. Moreover, attenuating mitochondrial-derived ROS would also indirectly attenuate additional ROS release from uncoupled eNOS (oxidation of $\mathrm{BH}_{4}$ ), Nox2 (ROS mediated cytokine release) and xanthine oxidase (ROS mediated microvascular dysfunction), thereby inhibiting all four known sources of ROS during reperfusion [18,20,31,41]. This is consistent with our findings in (Figures 3-6), which shows an inverse relationship between lowering blood $\mathrm{H}_{2} \mathrm{O}_{2}$ and increasing blood NO with both MitoQ and SS-31 treatment. Recently, Cai et al 2018 reported that SS-31 stabilized Superoxide Dismutase (SOD) and catalase levels determined by western blot in the hindlimb I/R model compared to control, which showed a relative decrease in SOD and catalase [25]. It is well known that SOD converts $\mathrm{SO}$ to $\mathrm{H}_{2} \mathrm{O}_{2}$ and catalase converts $\mathrm{H}_{2} \mathrm{O}_{2}$ to $\mathrm{H}_{2} \mathrm{O}$ to attenuate $\mathrm{ROS}$ production; therefore, the stabilizing effects of SS-31 on SOD and catalase activities in I/R injury would cause a further reduction of ROS [17,25]. Our results extend these findings and show that MitoQ and SS-31 treatment at the onset of reperfusion result in a decrease in blood $\mathrm{H}_{2} \mathrm{O}_{2}$ and an increase in blood NO compared to controls. Collectively, these new findings are consistent with our previous studies showing reduction of blood $\mathrm{H}_{2} \mathrm{O}_{2}$ and increased $\mathrm{NO}$ bioavailability in hindlimb I/R correlates with improved post-reperfused cardiac function and decreased infarct size in myocardial I/R [18,19,20,31,39].

\section{The role of mitochondria in $I / R$ injury}

Preserving the integrity of these energy-producing organelles is of vital importance to maintain cardiac function and reduce $I / R$ injury. It is well known that mitochondria are key sources of ROS, especially during the reperfusion phase of I/R [33]. Therefore, it is plausible that MitoQ and SS-31 act to scavenge mitochondrialderived ROS when given just during reperfusion. MitoQ is selectively concentrated within the mitochondria due to the large membrane potential gradient. It is reduced by the respiratory chain to ubiquinol, which is then oxidized to ubiquinone and accumulates in the mitochondria. Its antioxidant effects are recovered when ubiquinone is converted back to ubiquinol by the respiratory chain, which is active during reperfusion [13]. In contrast, the two positively charged amino acids of SS-31 enable this molecule to be concentrated in the mitochondria in an energy independent, non-saturable manner. This difference may be significant in that the mitochondrial membrane potential may be disrupted during reperfusion and therefore limit the effectiveness of MitoQ. Our results show that MitoQ (10 and 20 $\mu \mathrm{M})$ was cardioprotective when given upon reperfusion after a 30 min ischemic period, which indicates that either the mitochondrial membrane potential was sufficiently maintained despite $\mathrm{I} / \mathrm{R}$ or MitoQ can accumulate in the mitochondria despite dissipation of the mitochondrial membrane potential. Collectively, the data from both mitochondrial-directed agents would suggest that mitochondria are a major component of reperfusion-induced cell death, and consequently that attenuating mitochondrial-derived ROS at the beginning of reperfusion can mitigate the deleterious effects of ROS on infarct size and post-reperfused cardiac function.

\section{Future studies}

Considering that I/R induced ROS is a major component of reperfusion injury, inhibiting more than one major source of $I / R$ derived ROS may lead to even further reduction in infarct size. It is well known that mitochondrial dysfunction, NADPH oxidase, uncoupled eNOS, and xanthine oxidase are the principal sources of I/R derived ROS. Therefore, inhibiting mitochondrial-derived ROS combined with a Nox 2 inhibitor should be more effective in reducing the deleterious effects of reperfusion injury than either agent alone. Thus, it would be interesting to test a combination of a Nox 2 inhibitor (e.g., apocynin or Nox2ds peptide) and a mitochondrial antioxidant (e.g., MitoQ or SS-31) on post-perfused cardiac function, infarct size, and blood $\mathrm{H}_{2} \mathrm{O}_{2} / \mathrm{NO}$ in myocardial and hindlimb $\mathrm{I} / \mathrm{R}$, respectively. Such studies would help determine the extent of the contribution of ROS to I/R injury.

\section{References}

1. Hausenloy DJ, Yellon DM (2008) Time to take myocardial reperfusion injury seriously. N Engl J Med 359: 518-520.

2. Benjamin EJ, Blaha MJ, Chiuve SE, Cushman M, Das SR, et al. (2017) Heart Disease and stroke statistics-2017 update: a report from the american heart association. Circulation135: e146-e603.

3. Yellon DM, Hausenloy DJ (2007) Myocardial reperfusion injury. N Engl J Med 357: 1121-1135.

4. Sanada S, Komuro I, Kitakaze M (2011) Pathophysiology of myocardial reperfusion injury: preconditioning, postconditioning, and translational aspects of protective measures. Am J Physiol Heart Circ Physiol 301: $\mathrm{H} 1723-\mathrm{H} 1741$.

5. Bulluck H, Yellon DM, Hausenloy DJ (2016) Reducing myocardial infarct size: challenges and future opportunities. Heart 102: 341-348.

6. Ferdinandy P, Schulz R, Baxter GF (2007) Interaction of cardiovascular risk factors with myocardial ischemia/reperfusion injury, preconditioning, and postconditioning. Pharmacol Rev 59: 418-458.

7. Garcia-Dorado D, Ruiz-Meana M, Inserte J, Rodriguez-Sinovas A, Pipe HM (2012) Calcium-mediated cell death during myocardial reperfusion. Cardiovasc Res 94: 168-180.

8. Hausenloy DJ, Yellon, DM (2013) Myocardial ischemia-reperfusion injury: a neglected therapeutic target. J Clin Invest 123: 92-100.

9. Ruiz-Meana M, Abellan A, Miro-Casas E, Agullo E, Garcia-Dorado D (2009) Role of sarcoplasmic reticulum in mitochondrial permeability transition and cardiomyocyte death during reperfusion. Am J Physiol Heart Circ Physiol 297: $\mathrm{H} 1281-\mathrm{H} 1289$.

10. Ide T, Tsutsui $H$, Kinugawa S, Utsumi H, Kang D, et al. (1999) Mitochondrial electron transport complex $I$ is a potential source of oxygen free radicals in the failing myocardium. Circ Res 85: 357-363.

11. Muller FL, Liu Y, Van Remmen H (2004) Complex III releases superoxide to both sides of the inner mitochondrial membrane. J Biol Chem 279: 49064 49073. 
Citation: Patel H, Ondrasik R, Galbreath T, Lopez A, Walker S, et al. Mitochondrial-Targeted Antioxidants Given at Reperfusion Protect Cardiac and Hindlimb Muscles against Ischemia/Reperfusion Injury. J Cardiobiol. 2019;6(1): 8.

12. Orogo AM, Gustafsson AB (2013) Cell death in the myocardium: my heart won't go on. IUBMB Life 65: 651-656.

13. Adlam VJ, Harrison JC, Porteous CM, James AM, Smith RA, et al. (2005) Targeting an antioxidant to mitochondria decreases cardiac ischemiareperfusion injury. FASEB J 19: 1088-1095.

14. Szeto HH (2006) Cell-permeable, mitochondrial-targeted, peptide antioxidants. AAPS J 8: E277-E283.

15. Szeto HH (2006) Mitochondria-targeted peptide antioxidants: nove neuroprotective agents. AAPS J 8: E521-E531.

16. Lefer AM, Lefer DJ (1996) The role of nitric oxide and cell adhesion molecules on the microcirculation in ischaemia-reperfusion. Cardiovasc Res 32: 743 751

17. Schmidt TS, Alp NJ (2007) Mechanisms for the role of tetrahydrobiopterin in endothelial function and vascular disease. Clin Sci (Lond) 113: 47-63.

18. Perkins KA, Pershad S, Chen Q, McGraw S, Adams JS, et al. (2012) The effects of modulating eNOS activity and coupling in Ischemia/Reperfusion (I/R). Naunyn Schmiedebergs Arch Pharmacol 385: 27-38.

19. Chen Q, Kim EE, Elio K, Zambrano C, Krass S, et al. (2010) The role of tetrahydrobiopterin and dihydrobiopterin in ischemia/reperfusion injury when given at reperfusion. Adv pharmacol Sci 2010: 963914

20. Chen Q, Devine I, Walker K, Pham H, Ondrasik R, et al. (2016) Nox2ds Tat, a peptide inhibitor of NADPH oxidase, exerts cardioprotective effects by attenuating reactive oxygen species during ischemia/reperfusion injury. Am J Biomed Sci 8: 208-227.

21. Kelso GF, Porteous CM, Coulter CV, Hughes G, Porteous WK, et al. (2001) Selective targeting of a redox-active ubiquinone to mitochondria within cells: antioxidant and antiapoptotic properties. J Biol Chem 276: 4588-4596.

22. Chacko BK, Reily C, Srivastava A, Johnson MS, Ye Y, et al. (2010) Prevention of diabetic nephropathy in Ins2(+/)(-)(AkitaJ) mice by the mitochondriatargeted therapy MitoQ. Biochem J 432: 9-19.

23. Zhao K, Zhao GM, Wu D, Soong Y, Birk AV, et al. (2004) Cell-permeable peptide antioxidants targeted to inner mitochondrial membrane inhibit mitochondrial swelling, oxidative cell death, and reperfusion injury. J Bio Chem 279: 34682-34690.

24. Neuzil J, Widen C, Gellert N, Swettenham E, Zobalova R, et al. (2007) Mitochondria transmit apoptosis signalling in cardiomyocyte-like cells and isolated hearts exposed to experimental ischemia-reperfusion injury. Redox Rep 12: 148-162.

25. Cai J, Jiang Y, Zhang M, Zhao H, Li H, et al. (2018) Protective effects of mitochondrion-targeted peptide SS-31 against hind limb ischemia-reperfusion injury. J Physiol Biochem 74: 335-343.

26. Bell RM, Mocanu MM, Yellon DM (2011) Retrograde heart perfusion: the Langendorff technique of isolated heart perfusion. J Mol Cell Cardiol 50: 940-
27. McIntyre AA, Kim H, Woodley J, Dittakavi T, Finnegan M, et al. (2018) protein Kinase $\mathrm{C}$ epsilon peptide inhibitor exerts cardioprotective effects in myocardial Ischemia/Reperfusion injury. J Cardiobiol 5: 1-6.

28. Ondrasik R, Chen Q, Navitsky K, Chau W, Lau OS, et al. (2013) Cardioprotective effects of mitochondrial targeted antioxidants in myocardial Ischemia/Reperfusion (I/R) injury. Proceedings of the $23^{\text {rd }}$ American Peptide Symposium, American Peptide Society 64-65.

29. Galbreath T, Chen Q, Ondrasik R, Bertolet M, Barsotti R, et al. (2013) Effects of mitochondrial-targeted antioxidants on real-time nitric oxide and hydrogen peroxide release in hind limb Ischemia and Reperfusion (I/R). Proceedings of the $23^{\text {rd }}$ American Peptide Symposium, American Peptide Society 46-47.

30. Inagaki K, Hahn HS, Dorn GW 2nd, Mochly-Rosen D (2003) Additive protection of the ischemic heart ex vivo by combined treatment with delta-protein kinase C inhibitor and epsilon-protein kinase C activator. Circulation 108: 869-875.

31. Chen Q, Woodworth PC, Devine I, Ondrasik R, Habtamu T, et al. (2016) Apocynin exerts dose-dependent cardioprotective effects by attenuating reactive oxygen species in Ischemia/Reperfusion. Cardiovasc Pharm 5: 1-7.

32. Sutherland FJ, Hearse DJ (2000) The isolated blood and perfusion fluid perfused heart. Pharmacol Res 41: 613-627.

33. Cho J, Won K, Wu D, Soong Y, Liu S, et al. (2007) Potent mitochondriatargeted peptides reduce myocardial infarction in rats. Coron Artery Dis 18: 215-220.

34. Montgomery M, Adams JS, Teng JC, Biruk T, Ondrasik R, et al. (2013) Myristoylated protein kinase $C$ Epsilon peptide inhibitor exerts cardioprotective effects in rat and porcine myocardial ischemia/reperfusion: a translational research study. Proceedings of the Twenty third American and the Sixth international peptide symposium.

35. Szeto HH (2008) Mitochondria-targeted cytoprotective peptides for ischemiareperfusion injury. Antioxid Redox Signal 10: 601-619.

36. Roos ST, Timmers L, Biesbroek PS, Nijveldt R, Kamp O, et al. (2016) No benefit of additional treatment with exenatide in patients with an acute myocardial infarction. Int J Cardiol 220: 809-814

37. Cung TT, Morel O, Cayla G, Rioufol G, Garcia-Dorado D, et al. (2015) Cyclosporine before $\mathrm{PCl}$ in patients with acute myocardial infarction. $\mathrm{N}$ Engl J Med 373: 1021-1031.

38. Kloner RA, Hale SL, Dai W, Gorman RC, Shuto T, et al. (2012) Reduction of ischemia/reperfusion injury with bendavia, a mitochondria-targeting cytoprotective Peptide. J Am Heart Assoc 1: e001644.

39. Teng JC, Kay H, Chen Q, Adams JS, Grilli C, et al. (2008) Mechanisms related to the cardioprotective effects of protein kinase $\mathrm{C}$ epsilon (PKC epsilon) peptide activator or inhibitor in rat Ischemia/Reperfusion injury. Naunyn Schmiedebergs Arch Pharmacol 378: 1-15.

40. Frangogiannis NG, Lindsey ML, Michael LH, Youker KA, Bressler RB, et al. (1998) Resident cardiac mast cells degranulate and release preformed TNF-alpha, initiating the cytokine cascade in experimental canine myocardial ischemia/reperfusion. Circulation 98: 699-710.

41. Carden DL, Granger DN (2000) Pathophysiology of Ischemia-Reperfusion injury. J Pathol 190: 255-266.

\section{Acknowledgement}

We would like to acknowledge Jennifer Dang, Biomedical Sciences graduate student at PCOM, Arjun Nair, a Physician Scientist in Training student at PCOM, Tameka Dean and lan Madison, D.O. students at PCOM, for formatting the revised Figures and Figure legends and text throughout our manuscript. 